\title{
Immunopathogenesis of ophthalmological paraneoplastic syndromes: Recent findings
}

\author{
Monika Kordyśf,B-D,F, Joanna Przeździecka-Dołyk 2,3,A-D,F, Anna Turno-Kręcicka ${ }^{2, E, F}$, Marta Misiuk-Hojło2, 2,F \\ ${ }^{1}$ Student, Faculty of Medicine, Wroclaw Medical University, Poland \\ 2 Department and Clinic of Ophthalmology, Faculty of Postgraduate Medicine, Wroclaw Medical University, Poland \\ ${ }^{3}$ Department of Optics and Photonics, Faculty of Fundamental Problems of Technology, Wrocław University of Science and Technology, Poland \\ A - research concept and design; $\mathrm{B}$ - collection and/or assembly of data; $\mathrm{C}$ - data analysis and interpretation; \\ $\mathrm{D}$ - writing the article; $\mathrm{E}$ - critical revision of the article; $\mathrm{F}$ - final approval of the article
}

Address for correspondence

Joanna Przeździecka-Dołyk

E-mail:arie_l@wp.pl

\section{Funding sources}

Pbmn-168 (grant for Young Researchers funded

by Wroclaw Medical University), Poland

Conflict of interest

None declared

Received on November 24, 2016

Reviewed on April 8, 2017

Accepted on May 18, 2017

\begin{abstract}
The aim of this study was to summarize the current knowledge of paraneoplastic syndromes involving eyes. The main interest was the immunopathogenesis of the abovementioned entities. A web search was conducted using Medline, Web of Science and Scopus engines. Key words concerning ocular paraneoplastic syndromes (OPS) such as: "ocular paraneoplastic syndrome", "cancer-associated retinopathy", "cancer-associated cone dysfunction", "melanoma-associated retinopathy", "bilateral diffuse uveal melanocytic proliferation", and "paraneoplastic optic neuritis" were combined with "immunology", "immune response", "antibodies", or "autoantibodies". Numerous papers were found as a result of "ocular paraneoplastic syndrome" search and many of them matched the chosen criteria. We focused on the most recent papers - published in the last 5 years - and eventually, 92 items were found. Only several papers from each detailed category fulfilled both OPS and immunologic criteria. Site-specific paraneoplastic syndromes still remain a difficult clinical challenge. The immunopathogenesis of some of them seems to be more complex than previously thought.
\end{abstract}

Key words: ophthalmologic findings, optic neuropathy, immunopathogenesis, paraneoplastic syndrome, paraneoplastic retinopathy

DOI

$10.17219 /$ acem/73860

\section{Copyright}

Copyright by Author(s)

This is an article distributed under the terms of the

Creative Commons Attribution Non-Commercial License

(http://creativecommons.org/licenses/by-nc-nd/4.0/) 


\section{Introduction}

The incidence of paraneoplastic syndromes is estimated at $7-15 \%$ in patients suffering from malignant neoplasms, varying in different papers. ${ }^{1}$ However, the frequency of the eye site-specific manifestation is unknown. This disorder is caused by chemical substances (produced by neoplasm) that have an impact on distant tissues and organs, not simply by mass effect or metastatic infiltration. Sawyer et al. in 1976 were the first to describe the visual acuity loss caused by retinal degeneration connected with systemic cancer. Since that report, many patients have been described suffering from ocular paraneoplastic syndromes (OPS). The usual presented classification of OPS distinguishes paraneoplastic retinopathies, paraneoplastic optic neuropathy and paraneoplastic tonic pupils. Other authors divide OPS into those with visual loss and with abnormal eye movements, incorporating the last mentioned group of diseases as only ophthalmological.

The tumor during its neoplastic transformation can develop some antigens similar to those naturally occurring in different organs. Human immune system tries to protect the organism via humoral or cellular response against the tumor. This immunologic response plays an important role or is the main cause for the paraneoplastic syndromes occurring in patients suffering from neoplasms. Moreover, in organs and systems where the specific blood-tissue barriers can be found, the neoplastic transformation can lead to expressing proteins similar to those that can be found in restricted sites (Fig. 1). The described situation promotes an aggressive response of the patient's own immune system towards healthy tissues. In previous papers, the autoantibody against eye-specific proteins as well as rare infiltrates of $\mathrm{T}$ cells, plasma cells and macrophages in both retinal and cancer tissues were described.

The aim of this paper is to summarize the available knowledge about immunopathogenesis of OPS. The resources were searched using Medline, Web of Science, Scopus, PubMed, and worldwide websites. Searched terms included: "ocular paraneoplastic syndrome", "cancer-associated retinopathy", "cancer-associated cone dysfunction", "melanoma-associated retinopathy", "bilateral diffuse uveal melanocytic proliferation", and "paraneoplastic optic neuritis", combined with "immunology", "immune response", “T cell”, "B cell”, "innate lymphoid cell”, "antibodies", and "autoantibodies".

Numerous papers were found for "ocular paraneoplastic syndrome" - 299 items. We focused on the most recent papers, published within the last 5 years - in this case 92 items were found. Only a few papers from each category fitted both OPS and immunologic criteria.

\section{Cancer-associated retinopathy}

There is a theory that cancer-associated retinopathy (CAR) is primarily caused by $p 53$ tumor suppressor gene mutation in tumor cells, which express highly immunogenic recoverin protein. In response, the patient's immune system produces a high level of anti-recoverin

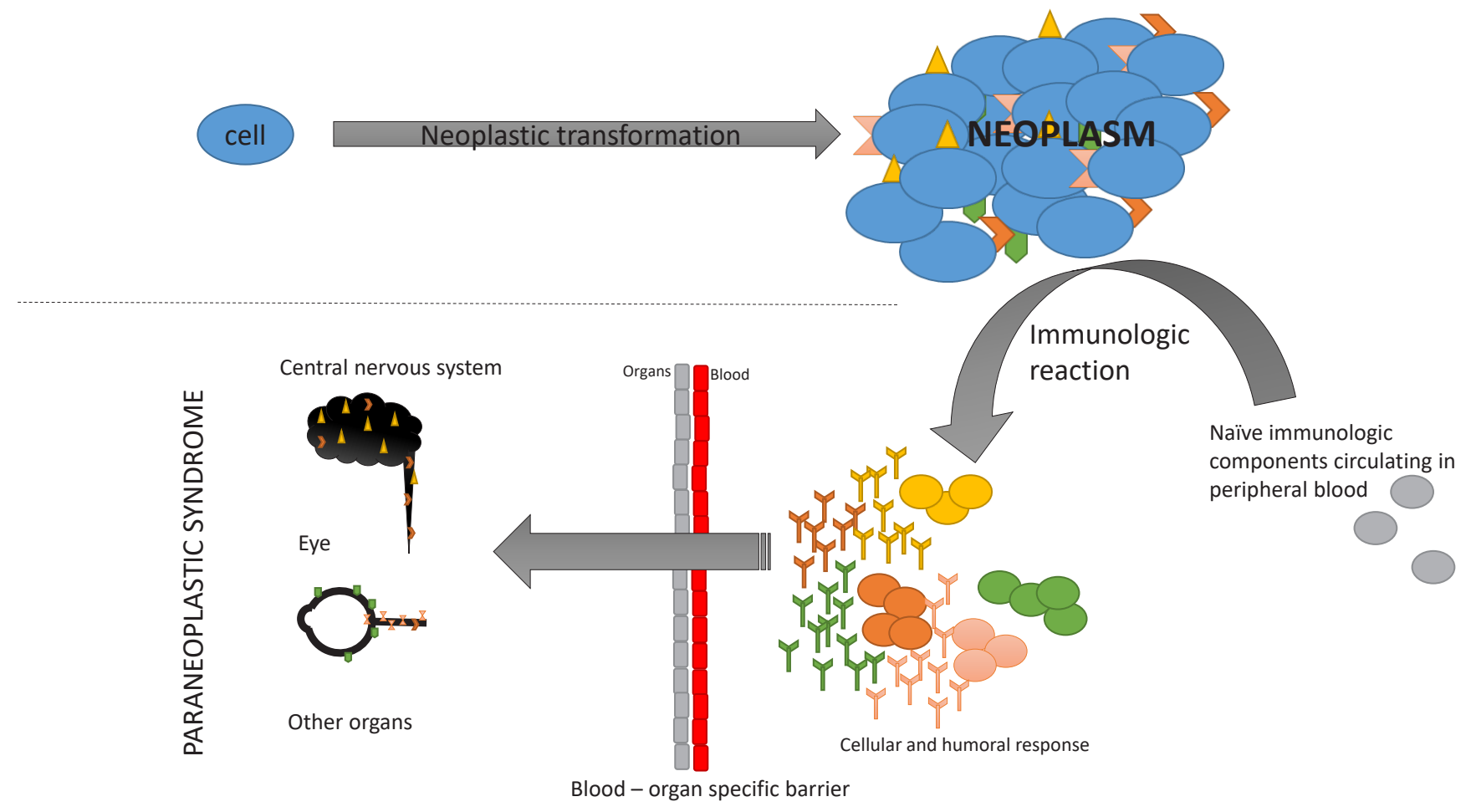

Fig. 1. Development of site-specific paraneoplastic syndrome. The importance of immunologic reactions in its pathogenesis 
IgG. It penetrates through the blood-retinal barrier and by binding to recoverin molecules on the photoreceptors cells, activates caspase-dependent apoptotic pathways. Immunological reaction is conducted via cytotoxic T-lymphocytes antigen $4(C T L A-4)$ and vascular endothelial growth factor (VEGF) receptor-1. In such cases, the recoverin-specific $\mathrm{T}$ cells including CD8+ lymphocytes were found in the peripheral blood. ${ }^{1}$ All the abovementioned changes lead to photoreceptor cell death. Rods and cones are equally affected; as a consequence, flattening of the alpha wave in electroretinogram (ERG) is observed. ${ }^{2,3}$

After the first description of recoverin, many other autoantigens that generate a clinical presentation similar to recoverin were discovered. ${ }^{1,2,4}$ According to some authors, different molecules transmitted via different pathways cause slightly different clinical symptoms. However, nowadays CAR is thought to be a group of clinically similar paraneoplastic retinopathies with a different molecular background that leads to photoreceptor death. ${ }^{1}$ Other antigens associated with CAR are: retinal enolase, Tubby-like protein 1 (TULP1), aryl hydrocarbon receptor interacting protein-like 1 (AIPL1), interphotoreceptor retinoid binding protein (IRBP), photoreceptor cell-specific nuclear receptor (PNR), glyceraldehyde 3-phosphate dehydrogenase $(G A P D H)$, aldolase, transducing- $\alpha$, as well as heat shock cognate protein 70 (hsc-70) and 60 (hsc-60). ${ }^{5-9}$ Additionally, some authors indicate other possible antigens that can be associated with CAR, including: guanylyl cyclase-activating proteins (GCAPs), heat shock protein 27 (HSP27), Rab6A GTPase (Rab6A), carbonic anhydrase II (CA II), anti-CV2/collapsin response mediator protein (CRMP5), and anti-Hu antibodies. ${ }^{7,10-13}$ Furthermore, Adamus et al. showed that CA II unique epitopes can be used to differentiate autoimmune retinopathies (AR) from CAR. Autoimmune retinopathies sera predominantly reacted with the N-terminal epitope 85-90 and CAR sera have been found to be reactive with the peptide 218-222 clustered within the $\alpha$-helix. ${ }^{10}$ Turaka et al. described a case of CAR with positive antiretinal autoantibodies against 48-kDa (arrestin), 64-kDa and 94-kDa, probably in the course of ovarian mass, in 14-year-old girl of African origin. ${ }^{3}$

Common clinical manifestations of the first ophthalmological symptoms include both cone dysfunction (photosensitivity, prolonged glare, decreased best-corrected visual acuity - BCVA, color discrimination, and central scotomas) and rode dysfunction (night blindness, prolonged adaptation to darkness and peripheral or ring scotomas). Other clinical symptoms have been described as arteriolar narrowing, retinal pigment epithelial thinning and motting, vitritis, cells in anterior chamber, sheathing of retinal arterioles, periphlebitis, and pallor of the optic disc. In ERG rods- and cones-mediated responses are not recordable or significantly decreased.

Neoplasms causing CAR can be as follows: smallcell carcinoma of the lung, other neoplasm of the lung, breast cancer, osteosarcoma, cancers of the cervix, ovary, uterus and thymus, Warthin tumor of parotid gland, as well as carcinoma of unknown origin. ${ }^{1-3,5-7,11,12}$ There are single reports of patients with prostate, pancreatic neurocrine and small intestine tumors. ${ }^{14,15}$ In the past CAR has been reported in bladder and laryngeal neoplasms as well as in lymphomas (systemic follicular cell lymphoma) or colon adenoma - only as case reports or case series. ${ }^{4,8}$

\section{Cancer-associated cone dysfunction}

Dysfunction of the cone system alone without any symptoms or electrophysiological signs of rods system failure has rarely been reported. ${ }^{16}$ Clinical symptoms include: mild to moderate BCVA loss, sudden photosensitivity, and total or subtotal loss of color perception. In addition, patients usually notice improvement of the visual acuity while wearing sunglasses. The ERG shows the suppression of cones response. . $^{17,18}$

Cancer-associated cone-specific dystrophy involves an abnormal amount of immunologic activity with 2 retinal antigens that are possibly isoforms of the same $40-\mathrm{kD}$ photoreceptor protein. ${ }^{19}$ This protein may be expressed only in cones, and perhaps more in $\mathrm{L}$ and $\mathrm{M}$ than $\mathrm{S}$ cones. ${ }^{17}$ The circulating antibodies in cancer-associated cone dysfunction (CACD) include the CAR antigen/recoverin and protein whose molecular weight is 50 and $40 \mathrm{kDa} .{ }^{19}$

Finger et al. described serum derived 42-kDa retinal antigen in a patient suffering from CACD and paraneoplastic optic neuropathy (PON). ${ }^{16}$

Paraneoplastic retinopathies also include the anti-enolase retinopathy that characteristically occurs together with cone dysfunction. In enolase-associated autoimmune retinopathy, visual impairment and the course of the disease may vary from relative stability to slow progression with loss of central vision. ${ }^{19}$

Reported cases of the abovedescribed paraneoplastic syndrome were associated with small-cell endometrial cancer, primary cervical intraepithelial neoplasia and laryngeal carcinoma. ${ }^{18,19}$

\section{Melanoma-associated retinopathy}

Patients suffering from melanoma can develop paraneoplastic retinopathy caused by pure dysfunction of rods without any cone involvement. Clinical symptoms may include: sudden shimmering, flickering, difficulty with the night vision, and photopsias (pulsating, continuous or intermittent) with occasional hyperphotosensitivity and floaters. Symptoms usually are observed in patients already treated with melanoma and the development of melanoma-associated retinopathy (MAR) is considered 
to be a bad prognosis marker. Lesions are usually bilateral with the time-shift between the eyes from weeks to months. Typically unaffected, BCVA, visual field and color vision can, however, be deteriorated in rare cases (e.g., generalized depression in flicker perimetry and redgreen axis or blue-yellow axis demonstrated with Farnsworth-Munsell 100 Hue Color Vision Test). In the fundus examination, of the disc pallor, non-specific retinal pigment changes or the attenuated retinal vessels can be found. The ERG findings are similar to those associated with congenital stationary night blindness: markedly reduced or absent dark-adapted b-wave with normal a-wave ("a negative-appearing waveform"), preserved or slightly reduced amplitude of light-adapted response, as well as prolonged implicit time and amplitude reduction of oscillatory potentials. It has been proven that some changes can be restricted only to the magnocellular pathway. ${ }^{20}$

Recently, MAR-like retinopathies have been described. ${ }^{21-23}$ Aronow et al. in 2012 described paraneoplastic vitelliform retinopathy (PVtR). ${ }^{23}$ In reported cases of PVtR, the average age of patients was 56 years at the moment of onset, equally distributed between males and females. The majority had metastatic cutaneous melanoma. Paraneoplastic vitelliform retinopathy is characterized by subjective visual loss with no BCVA reduction, nyctalopia and photopsias. Funduscopic findings vary among reporting authors from bilateral serous macular detachments, "vitelliformpseudohypopyon macular appearance" to multiple yellow and white lesions scattered throughout the posterior pole and mid-periphery. ${ }^{22}$ Aronow et al. described nummular, flat and well-demarcated lesions that clinically appear to be located within the deep retinal layers. ${ }^{23}$ However, ERG recordings showed reduction in the b-wave amplitude, which implies that there is selective destruction of bipolar cells. In the case presented by Aronow et al., ERG revealed a mild reduction in both the a-wave and the b-wave amplitudes for both scotopic and photopic waveforms - it shows involvement of both rods and cones. Electrooculography (EOG) demonstrated a pathologically reduced Arden ratio (reflecting retinal pigment epithelium (RPE) dysfunction) only in $40 \%$ of the patients that have been examined (5 cases evaluated with EOG). ${ }^{23}$ Lincoff et al. described an exudative polymorphous vitelliform retinopathy case of 65 -year-old male suffering from metastatic melanoma with little subjective ocular-related complaints. ${ }^{21}$ This patient has been treated successfully with ipilimumab, an anti-cytotoxic T lymphocyte-associated antigen 4 antibody. Metastatic lesions, as well as the exudative macular lesions, improved with the administered treatment within 1 year of follow-up. ${ }^{21}$

In the sera of patients suffering from MAR and MAR-like retinopathies, specific autoantibodies against human rod bipolar cells can be found. ${ }^{24}$ Wang et al. presented postmortem paraneoplastic retinopathy as a cross-reaction of cutaneous metastatic melanoma autoantibodies. ${ }^{22,24}$
Authors reported local retinal thinning with the damaged inner nuclear layer and outer plexiform layer. Those changes resulted in focal retinal degeneration, edema and atrophy. There were no signs of infiltration of immunespecific or melanoma cells. Immunohistochemical reactions showed evidence of melanoma-associated autoantibodies directed against transient receptor potential M1 channels that target the ON-bipolar cell structures in the inner nuclear and outer plexiform layers in PVtR. ${ }^{22}$ Several cases of melanoma-associated retinopathy have been reported with serum autoantibodies to transient receptor potential cation channel, subfamily $M$, member 1 (TRPM1) labeled on ON-bipolar cells. ${ }^{20,25,26}$

Other autoantibodies similar to those associated with CAR can be found in MAR and MAR-like retinopathies, such as: autoantibodies directed against $\alpha$-enolase, recoverin or hsc-70; autoantibodies specific for bipolar cells and non-specific for bipolar cells; carbonic anhydrase II (CA II), interphotoreceptor retinoid binding protein (IRBP), bestrophin, myelin basic protein, and rod outer segment proteins have been reported. ${ }^{23}$

Munk et al. described the case of a reversible nyctalopia in a 70-year-old patient with lung adenocarcinoma diagnosed 8 months previously, who has been enrolled in a phase I trial with erlotinib hydrochloride combined with AUY922. The extensive loss of the ellipsoid zone as well as external limiting membrane was shown in spectral domain optical coherence tomography (SD-OCT) with the intact central zone (4 regular layers). The ERG findings included extinguished responses to blue scotopic light and significantly decreased responses in different light conditions. The anti- $\alpha$-enolase was found in the serum. Seventeen months after the cessation of AUY922 treatment the symptoms significantly improved, but there were still detectable irregularities within the ellipsoid zone and external limiting membrane in SD-OCT. ${ }^{27}$

Audemard et al. described a treatment option in the patients not responding to classical surgery and chemotherapy with metastatic melanoma presenting MAR. ${ }^{24}$ The ipilimumab has been reported previously to improve survival in previously treated patients with metastatic melanoma and with MAR-like PVtR. ${ }^{21}$ On the other hand, ipilimumab has not been recommended in the patients suffering from MAR at this same time due to the ability of the drug to increase autoimmunity. In the presented case of 70-year-old woman, the ipilimumab treatment enabled to free patient from the metastases (2-year observation) but exacerbated her vitiligo as well as MAR. ${ }^{24}$

Serous retinopathy-associated mitogen-activated protein kinase inhibition (binimetinib) for metastatic cutaneous and uveal melanoma has been reported recently by van Dijk et al. ${ }^{28}$ It is a time-dependent, reversible retinopathy with mild and transient visual symptoms. Treatment with binimetinib may be a cause of the described serous retinopathy; however, authors consider autoantibodies role in the pathogenesis of this entity. 


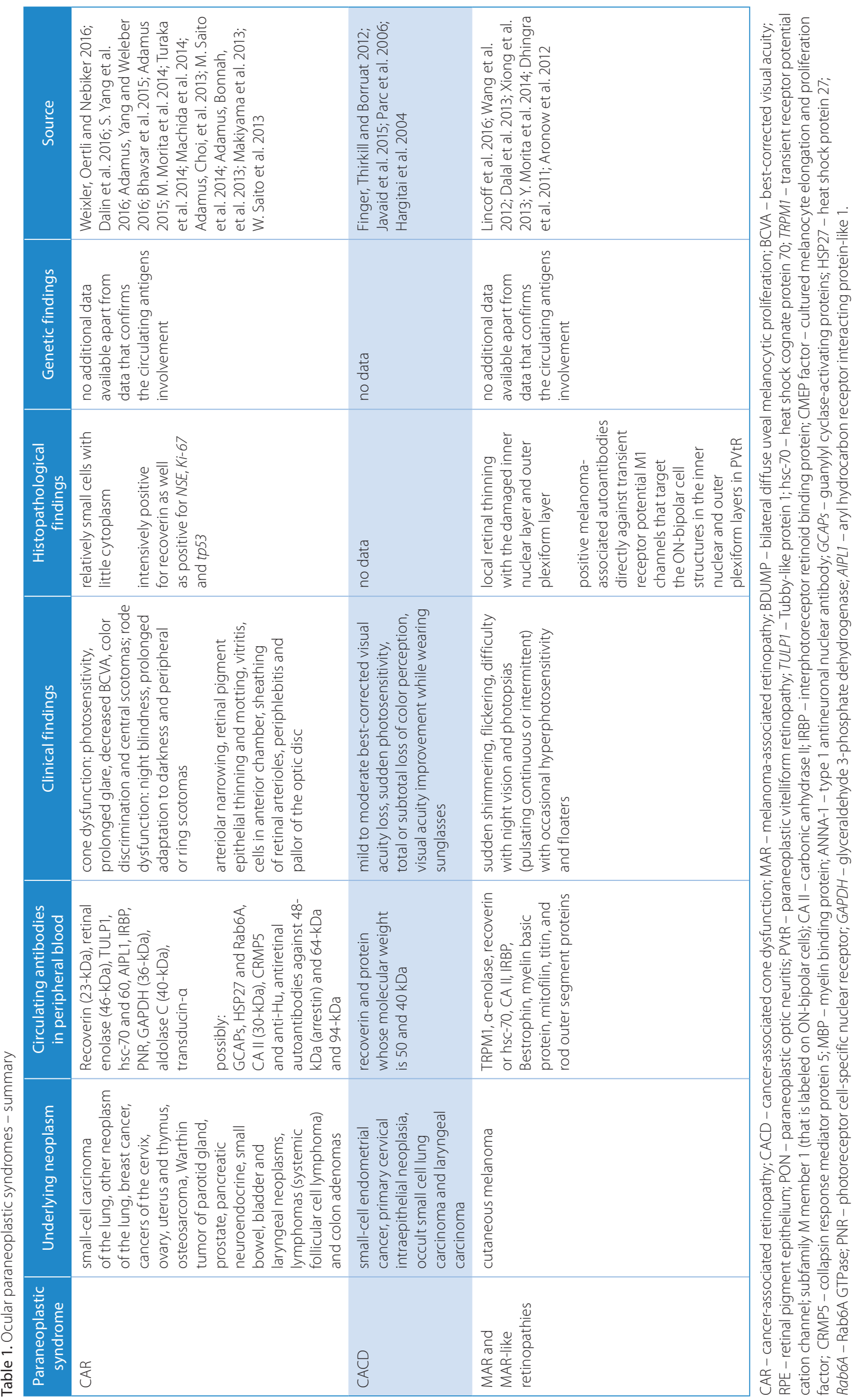




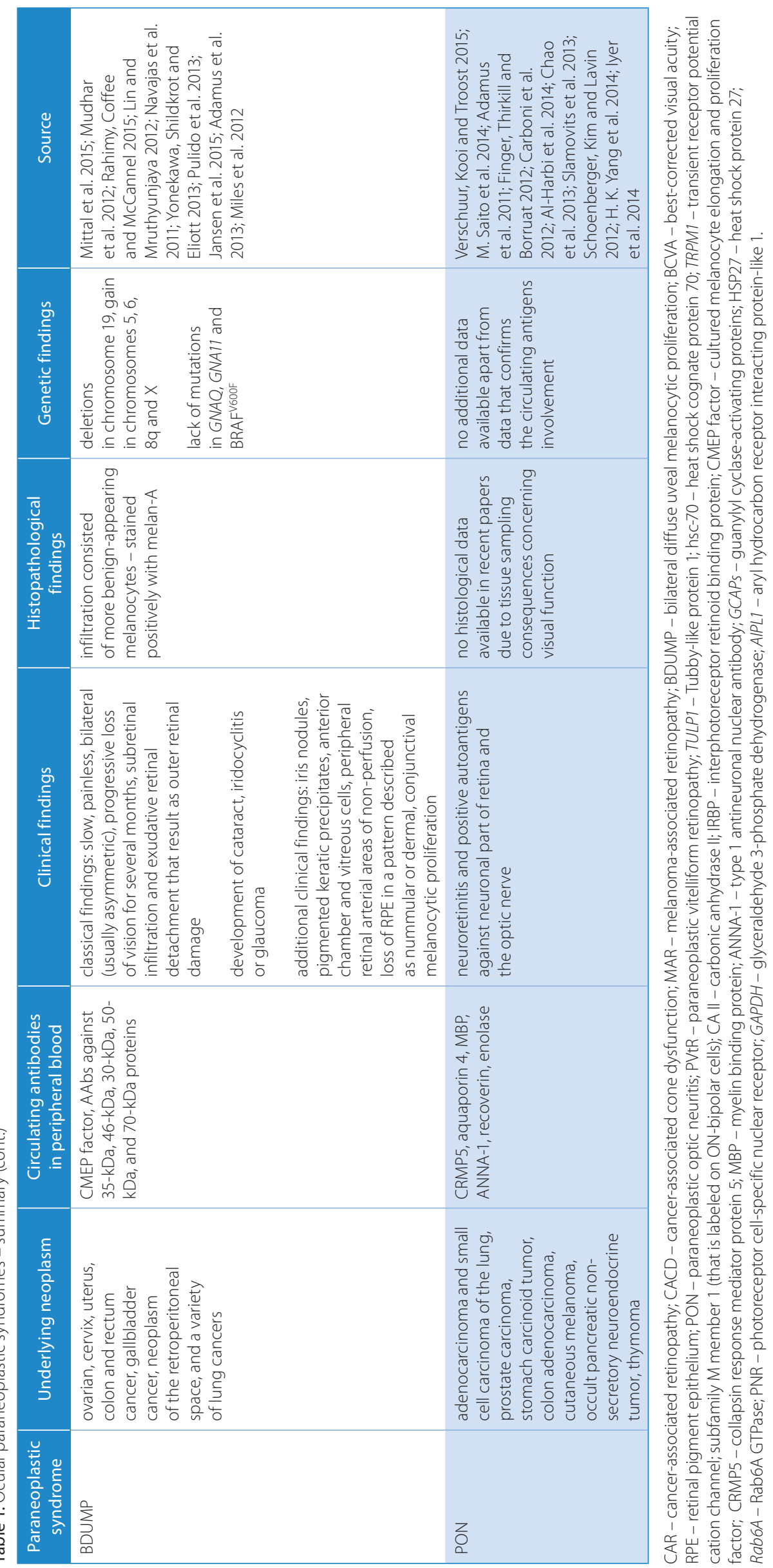




\section{Bilateral diffuse uveal melanocytic proliferation}

In the first reports describing diffuse uveal melanocytic proliferation (noted in the 1960s by Machemer and named in the 1980s by Barr et al.), the findings were thought to be a result of infiltration of the choroid by malignant ocular melanoma or choroidal metastases from cutaneous malignant melanoma. The later histopathologic findings revealed that the infiltration is composed by more benign-appearing melanocytes. The cellular infiltration, not the degeneration as in previously described conditions (CAR, MAR and cancerassociated cone dysfunction), is responsible for symptoms and findings that characterize the bilateral diffuse uveal melanocytic proliferation (BDUMP). ${ }^{29}$

The pathogenesis of the BDUMP is unknown due to its rare presentation and there is no consensus of whether it is a neoplastic, hyperplastic or paraneoplastic process. Three main mechanisms have been proposed: 1 . unknown carcinogenic factor that simultaneously inducts primary neoplasm and melanocytic proliferation development; 2. BDUMP is secondary to the secreted by cancer (primary cause) hormone or factor; 3 . genetic predisposition that causes coincidental development of the BDUMP and extraocular neoplasm. Interestingly, the melanocytic proliferation can be induced ex vivo in cultured melanocytes by adding the IgG fraction of human serum (by some authors named as cultured melanocyte elongation and proliferation factor - CMEP factor) from patients with BDUMP and the proliferation of melanocytes usually reveals regression after total excision of primary neoplasm..$^{30}$ In a clinical diagnosis of BDUMP, the elongation and selective proliferation of cultured human melanocytes, if cultured with patients serum, has been confirmed. ${ }^{30,31}$ Positive visual as well as ophthalmoscopic response to plasmapheresis have been described. ${ }^{31-34}$ Recently, Jansen et al. demonstrated a relation between the level of serum CEMP factor (during treatment with plasmapheresis in conjunction with radiation and chemotherapy) and the proliferation of cultured human melanocytes. ${ }^{31}$ Adamus et al. described 2 patients presented with BDUMP in the course of gynecological cancers that had autoantibodies against $35-\mathrm{kDa}, 46-\mathrm{kDa}, 30-\mathrm{kDa}$, $50-\mathrm{kDa}$ and $70-\mathrm{kDa}$ proteins in serum. ${ }^{12}$ In addition, the lack of the mutation in genes GNAQ, GNA11 and BRAF $F^{V 00 F}$ supports the thesis of BDUMP being a part of the neoplastic process and probably different oncogenic pathway to uveal nevi and the majority of uveal melanoma. ${ }^{29,35,36}$

Patients suffering from BDUMP experience slow, painless, bilateral (usually asymmetric), progressive loss of vision for several months. The melanocytic proliferation leads to subretinal infiltration and exudative retinal detachment as a result of outer retinal damage. Visual acuity can also deteriorate due to the development of cataract, iridocyclitis or glaucoma. ${ }^{29}$ Gass et al. described the main features of the BDUMP: multiple round or oval red spots of patches at the level of retinal pigment epithelium (found in the posterior pole), multifocal hyperfluorescence during fluorescein angiography corresponding to the lesions, diffuse thickening of the uveal tract with multiple elevated pigmented and non-pigmented uveal melanocytic tumors, exudative retinal detachment (a late finding), and rapidly progressive cataract formation. ${ }^{37}$ In recent years, the increasing number of described cases adds to our knowledge of the BDUMP. Additional clinical findings are: iris nodules, pigmented keratic precipitates, anterior chamber and vitreous cells, peripheral retinal arterial nonperfusion, loss of RPE in a pattern described as nummular or dermal, and conjunctival melanocytic proliferation. ${ }^{34,38-40}$ Electrophysiological findings are non-specific to the syndrome and may involve reduction of amplitude of scotopic or photopic response. The systemic findings (apart those associated to the neoplasm itself) usually do not occur; however, in rare cases there have been described hyperpigmented lesions of the oral mucosa, penis and skin.

The pathologic spectrum of the BDUMP varies between patients. The infiltrating cells can be small and spindleshaped as well as more rounded or even epithelioid with homogenous dark-staining pattern. The described cells that infiltrate RPE, stain with S-100 protein and they contain pigment granules of various density, with rare mitotic figures. In rare cases, the cells can develop more malignant-appearing epithelioid cells that suggest the malignant melanocytic transformation. Often the melanophages are seen in the area of necrosis and the photoreceptors with retinal pigment epithelium show signs of degeneration.

The BDUMP is associated with a number of carcinomas: cervix, uterus, clear-cell carcinoma of the endometrium, colon and rectum cancer, gallbladder cancer, malignancy of the retroperitoneal space, as well as a variety of lung cancers. $^{29,37,41-43}$ The described ocular paraneoplastic syndrome precedes identification of the malignancy in $1 / 2$ or $2 / 3$ of cases (even by a few years). In patients with diagnosed malignancy, the BDUMP usually develops within 1 year.

\section{Paraneoplastic optic neuropathy}

Paraneoplastic retinopathy including CAR and PON are autoimmune diseases in which the host response to tumor antigens triggers cross-reactions to an overlapping epitope in the retina and/or the optic nerve. Depending on whether the syndrome is targeting the retina, optic nerve or both, the pathogenesis of these syndromes is poorly elucidated and may be characterized by progressive loss of visual acuity and/or visual field sensitivity, loss photoreceptors and/or retinal ganglion cells (RGCs) and their axons, and optic nerve head changes, such as pallor, hyperemia, edema, swelling, or dropout of the retinal nerve fiber layer (RNFL) by optic coherence tomography (OCT) imaging.

Patients diagnosed with paraneoplastic or non-paraneoplastic retinopathy often present with optic nerve problems not related to glaucoma. The perceived pathology 
of autoimmune optic neuropathy and glaucoma is different, although both might have a similar underlying autoimmune cause.

Paraneoplastic optic neuropathy - called by some authors "optic neuritis associated with neoplasm" - can be characterized by funduscopic features of neuroretinitis and positive autoantigens to neuronal part of retina and optic nerve. ${ }^{13}$ As for autoantigens causing PON, collapsin response mediator protein (CRMP5) has been most frequently reported. ${ }^{44}$ Patients with anti-CRMP5 antibodypositive PON generally develop funduscopic features of neuroretinitis. ${ }^{44}$ The pathogenesis of neuroretinitis is regarded as vasculitis at the optic disc. ${ }^{45}$

Autoimmunity might significantly influence the outcome of optic nerve degenerative process but the pathogenic process is not fully elucidated. For example, autoantibodies (AAbs) can exert their pathogenic effects if they gain access from the periphery to the central nervous system (CNS) or the eye when the integrity of the blood barriers is compromised.

Taking into account a large variety of possibly involved cells, such as RGCs, Muller cells, bipolar cells, amacrine cells, and, on the other hand optic nerve head as a part of neuronal system, this results in large variety of autoantigens.

The major optic nerve antigenic targets for patient's auto-antibodies can be divided into 4 categories as follows: (1) classical glycolytic enzymes involved in energy production, including $\alpha$ and $\gamma$ enolases, glyceraldehyde 3-phosphate dehydrogenase, which also reacted with retinal antigens; (2) neuronal-specific myelin proteins (myelin basic protein - MBP, myelin oligodendrocyte glycoprotein - MOG) or type 1 antineuronal nuclear antibody (ANNA-1) ${ }^{46}$; (3) collapsin response mediator protein 5 $(\mathrm{CRMP} 5)^{47}$; and (4) aquaporin-4 (AQP4). ${ }^{46,48,49}$

Also, other antibodies labeled as optic nerve components, such as axons, astrocytes and oligodendrocytes are present, but their role in the pathogenicity of the optic neuropathy needs to be evaluated further. The identity of cellular targets is important in better understanding the etiology of autoimmune retinopathy associated with optic neuropathies, either with or without retinopathy, and for developing better treatments.

Paraneoplastic optic neuropathy is associated with number of carcinomas such as adenocarcinoma and small cell of the lung, prostate carcinoma, stomach carcinoid tumor, colon adenocarcinoma, cutaneous melanoma, occult pancreatic non-secretory neuroendocrine tumor, and thymoma. ${ }^{13,16,44,46,50-55}$

\section{Conclusions}

Ophthalmological neoplastic syndromes can be associated with a wide range of neoplasms. Large variety of clinical findings as well as different autoantibody targets in each condition makes the differential diagnosis more difficult.
The recent advancements in this field should be widely used to improve the patient-centered health care. However, basing only on the retinal autoantibody results without proper history-taking procedure and clinical evaluation is inappropriate.

\section{References}

1. Adamus G. Latest updates on antiretinal autoantibodies associated with vision loss and breast cancer. Invest Ophthalmol Vis Sci. 2015;56(3):1680-1688.

2. Machida S, Ohguro H, Ishida K, Suzuki M, Kawagishi K. Recoverinassociated retinopathy secondary to Warthin tumor of parotid gland. Doc Ophthalmol. 2014;129(2):123-128.

3. Turaka K, Kietz D, Krishnamurti L, et al. Carcinoma-associated retinopathy in a young teenager with immature teratoma of the ovary. J AAPOS. 2014;18(4):396-398.

4. Saito W, Kase S, Ohguro H, Ishida S. Autoimmune retinopathy associated with colonic adenoma. Graefes Arch Clin Exp Ophthalmol. 2013;251(5):1447-1449.

5. Dalin F, Adamus G, Yang S, et al. Aryl hydrocarbon receptor-interacting protein-like 1 in cancer-associated retinopathy. Ophthalmology. 2016;123(6):1401-1404.

6. Weixler B, Oertli D, Nebiker CA. Cancer-associated retinopathy as the leading symptom in colon cancer. Clin Case Rep. 2016;4,(2):171-176.

7. Yang S, Dizhoor A, Wilson DJ, Adamus G. GCAP1, Rab6, and HSP27: Novel autoantibody targets in cancer-associated retinopathy and autoimmune retinopathy. Trans/ Vis Sci Technol. 2016;5(3):1.

8. Bhavsar KV, Hedges T, Thirkill CE, Reichel E. Paraneoplastic retinopathy associated with systemic follicular cell lymphoma. Ophthalmic Surg Lasers Imaging Retina. 2015;46(3):373-376.

9. Adamus G, Bonnah R, Brown L, David L. Detection of autoantibodies against heat shock proteins and collapsin response mediator proteins in autoimmune retinopathy. BMC Ophthalmol. 2013;13:48.

10. Adamus G, Yang S, Weleber RG. Unique epitopes for carbonic anhydrase II autoantibodies related to autoimmune retinopathy and cancer-associated retinopathy. Exp Eye Res. 2016;147:161-168.

11. Morita M, Fukuhara T, Takahashi H, Maemondo M. Small cell lung cancer and progressive retinopathy. BMJ Case Rep. 2014;2014. doi: 10.1136/bcr-2014-205888

12. Adamus G, Choi D, Raghunath A, Schiffman J. Significance of antiretinal autoantibodies in cancer-associated retinopathy with gynecological cancers. J Clin Exp Ophthalmol. 2013;4(6):307.

13. Saito M, Saito W, Kanda A, Ohguro H, Ishida S. A case of paraneoplastic optic neuropathy and outer retinitis positive for autoantibodies against collapsin response mediator protein-5, recoverin, and a-enolase. BMC Ophthalmol. 2014;14:5.

14. Ogra S, Sharp D, Danesh-Meyer H. Autoimmune retinopathy associated with carcinoid tumor of the small bowel. J Clin Neurosci. 2014;21(2):358-360.

15. Y. Makiyama, Kikuchi T, Otani A, et al. Clinical and immunological characterization of paraneoplastic retinopathy. Invest Ophthalmol Vis Sci. 2013;54(8):5424-5431.

16. Finger ML, Thirkill CE, Borruat FX. Unusual paraneoplastic cause of vision loss: Combined paraneoplastic cone dystrophy and optic neuropathy. Arch Ophthalmol. 2012;130(5):660-662.

17. Hargitai J, MacKay C, Behrens M, Odel JG, Hood DC, Gouras P. Autoimmune-like cone dystrophy. Doc Ophthalmol. 2004;109(3):215-221.

18. Javaid Z, Rehan SM, Al-Bermani A, Payne G. Unilateral cancer-associated retinopathy: A case report. Scott Med J. 2016;61(3):155-159.

19. Parc CE, Azan E, Bonnel S, Sahel JA, Kaplan J, Thirkill CE. Cone dysfunction as a paraneoplastic syndrome associated with retinal antigens approximating 40 KiloDalton. Ophthalmic Genet. 2006;27(2):57-61.

20. Morita Y, Kimura K, Fujitsu Y, et al. Autoantibodies to transient receptor potential cation channel, subfamily $M$, member 1 in a Japanese patient with melanoma-associated retinopathy. Jpn J Ophthalmol. 2014;58(2);166-171.

21. Lincoff N, Nadeem M, Younus Z, Thirkill CE. Exudative polymorphous vitelliform retinopathy: Importance of early recognition of the condition in patients with metastatic melanoma. Ophthalmol Ther. 2016;5(1):121-127. 
22. Wang Y, Abu-Asab M, Li W, Aronow ME, Singh AD, Chan C-C. Autoantibody against transient receptor potential $M 1$ cation channels of retinal ON bipolar cells in paraneoplastic vitelliform retinopathy. BMC Ophthalmol. 2012;12:56.

23. Aronow ME, Adamus G, Abu-Asab M, et al. Paraneoplastic vitelliform retinopathy: Clinicopathologic correlation and review of the literature. Surv Ophthalmol. 2012;57(6):558-564.

24. Audemard A, de Raucourt S, Miocque S, et al. Melanoma-associated retinopathy treated with ipilimumab therapy. Dermatology. 2013;227(2):146-149.

25. Xiong W-H, Duvoisin RM, Adamus G, Jeffrey BG, Gellman C, Morgans CW. Serum TRPM1 autoantibodies from melanoma associated retinopathy patients enter retinal on-bipolar cells and attenuate the electroretinogram in mice. PloS One. 2013;8(8):e69506.

26. Dalal MD, Morgans CW, Duvoisin RM, et al. Diagnosis of occult melanoma using transient receptor potential melastatin 1 (TRPM1) autoantibody testing: A novel approach. Ophthalmology.2013;120(12):2560-2564.

27. Munk MR, Fernandes J, Mets M, Patel JD, Johnson ML, Jampol LM. Reversible nyctalopia and retinopathy in a patient with metastatic cancer treated with anti-heat shock protein 90 therapy. JAMA Ophthalmol. 2014;132(7):899-901.

28. van Dijk EH, van Herpen CM, Marinkovic M, et al. Serous retinopathy associated with mitogen-activated protein kinase inhibition (binimetinib) for metastatic cutaneous and uveal melanoma. Ophthalmology. 2015;122(9):1907-1916.

29. Mittal R, Cherepanoff S, Thornton S, Kalirai H, Damato B, Coupland SE. Bilateral diffuse uveal melanocytic proliferation: Molecular genetic analysis of a case and review of the literature. OculOncol Pathol.2015;2(2):94-99.

30. Miles SL, Niles RM, Pittock S, et al. A factor found in the IgG fraction of serum of patients with paraneoplastic bilateral diffuse uveal melanocytic proliferation causes proliferation of cultured human melanocytes. Retina. 2012;32(9):1959-1966.

31. Jansen JCG, van Calster J, Pulido JD, et al. Early diagnosis and successful treatment of paraneoplastic melanocytic proliferation. $\mathrm{Br} \mathrm{J}$ Ophthalmol. 2015;99(7):943-948.

32. Mets RB, Golchet $P$, Adamus $G$, et al. Bilateral diffuse uveal melanocytic proliferation with a positive ophthalmoscopic and visual response to plasmapheresis. Arch Ophthalmol. 2011;129(9):1235-1238.

33. Jaben EA, Pulido JS, Pittock S, Markovic S, Winters JL. The potential role of plasma exchange as a treatment for bilateral diffuse uveal melanocytic proliferation: A report of two cases. $J$ Clin Apher. 2011;26(6):356-361.

34. Pulido JS, Flotte TJ, Raja $\mathrm{H}$, et al. Dermal and conjunctival melanocytic proliferations in diffuse uveal melanocytic proliferation. Eye (Lond). 2013;27(9):1058-1062.

35. Mudhar HS, Scott I, UI-Hassanet A, et al. Bilateral diffuse uveal melanocytic hyperplasia: Molecular characterization and novel association with bilateral renal papillary carcinoma. Histopathology. 2012;61(4):751-754.

36. Rahimy E, Coffee RE, McCannel TA. Bilateral diffuse uveal melanocytic proliferation as a precursor to multiple systemic malignancies. Semin Ophthalmol. 2015;30(3):206-209.

37. Gass JD, Gieser RG, Wilkinson CP, Beahm DE, Pautler SE. Bilateral diffuse uveal melanocytic proliferation in patients with occult carcinoma. Arch Ophthalmol. 1990;108(4):527-533.

38. Lin P, Mruthyunjaya P. Retinal manifestations of oncologic and hematologic conditions. Int Ophthalmol Clin. 2012;52(1):67-91.

39. Navajas EV, Simpson ER, Krema H, et al. Cancer-associated nummular loss of RPE: Expanding the clinical spectrum of bilateral diffuse uveal melanocytic proliferation. Ophthalmic Surg Lasers Imaging. 2011;42:e103-106.

40. Yonekawa $Y$, Shildkrot $Y$, Eliott D. Inferior peripheral nonperfusion in bilateral diffuse uveal melanocytic proliferation. Ophthalmic Surg Lasers Imaging Retina. 2013;44(2):190-192.

41. Barr CC, Zimmerman LE, Curtin VT, Font RL. Bilateral diffuse melanocytic uveal tumors associated with systemic malignant neoplasms: A recently recognized syndrome. Arch Ophthalmol. 1982;100(2):249-255.

42. Margo CE, Pavan PR, Gendelman D, Gragoudas E. Bilateral melanocytic uveal tumors associated with systemic non-ocular malignancy: Malignant melanomas or benign paraneoplastic syndrome? Retina. 1987;7(3):137-141.

43. Leys AM, Dierick HG, Sciot RM. Early lesions of bilateral diffuse melanocytic proliferation. Arch Ophthalmol. 1991;109(11):1590-1594.
44. Adamus G, Brown L, Schiffman J, lannaccone A. Diversity in autoimmunity against retinal, neuronal, and axonal antigens in acquired neuro-retinopathy. J Ophthalmic Inflamm Infect. 2011;1(3):111-121.

45. Purvin V, Sundaram S, Kawasaki A. Neuroretinitis: Review of the literature and new observations. J Neuroophthalmol. 2011;31(1):58-68.

46. Yang HK, Woo SJ, Park W-Y, Hwang J-M. Paraneoplastic neuromyelitis optica associated with ANNA-1 antibodies in invasive thymoma. BMC Ophthalmol. 2014;14:106.

47. Rosencher L, Maisonobe T, Lavole A, Milleron B, Ferroir J-P. Neurologic paraneoplastic syndrome with anti-CV2/CRMP5 antibodies revealing a small cell lung cancer: Effectiveness of the lung cancer treatment [in French]. Rev Neurol (Paris). 2012;168(4):371-374.

48. M. Figueroa, Guo Y, Tselis A, et al. Paraneoplastic neuromyelitis optica spectrum disorder associated with metastatic carcinoid expressing aquaporin-4. JAMA Neurol. 2014;71(4):495-498.

49. Iyer A, Elsone L, Appleton R, Jacob A. A review of the current literature and a guide to the early diagnosis of autoimmune disorders associated with neuromyelitis optica. Autoimmunity. 2014;47(3):154-161.

50. Verschuur CVM, van der Kooi AJ, Troost D. Anti-aquaporin 4 related paraneoplastic neuromyelitis optica in the presence of adenocarcinoma of the lung. Clin Neuropathol. 2015;34(4):232-236.

51. Carboni G, Forma G, Bond AD, Adamus G, lannaccone A. Bilateral paraneoplastic optic neuropathy and unilateral retinal compromise in association with prostate cancer: A differential diagnostic challenge in a patient with unexplained visual loss. Doc Ophthalmol. 2012;125(1):63-70.

52. Al-Harbi T, Al-Sarawi A, Binfalah M, Dermime S. Paraneoplastic neuromyelitis optica spectrum disorder associated with stomach carcinoid tumor. Hematol Oncol Stem Cell Ther. 2014;7(3):116-119.

53. Chao D, Chen WC, Thirkill CE, Lee AG. Paraneoplastic optic neuropathy and retinopathy associated with colon adenocarcinoma. Can J Ophthalmol. 2013;48(5):e116-120.

54. Slamovits TL, Posner JB, Reidy DL, Thirkill CE, Keltner JL. Pancreatic neuroendocrine paraneoplastic optic neuropathy: Confirmation with antibody to optic nerve and hepatic metastasis. J Neuroophthalmol. 2013;33(1):21-25.

55. Schoenberger SD, Kim SJ, Lavin P. Paraneoplastic optic neuropathy from cutaneous melanoma detected by positron emission tomographic and computed tomographic scanning. Arch Ophthalmol. 2012;130(9):1223-1225. 